\title{
Editorial
}

\section{Misconceptions about brands}

There are three common misconceptions about brands that inhibit many efforts to build brands to their full potential. These misconceptions lead to what I label as the brand-is-an-island trap, the attribute fixation trap and the external perspective trap. Addressing these misconceptions is largely the motivation for writing my latest book 'Building Strong Brands'.

The brand-is-an-island trap follows from assuming that brands such as Hewlett-Packard or Wells Fargo or AT\&T can and should be managed in isolation. In fact, virtually all brands are part of a larger system consisting of many intertwined and overlapping brands and subbrands. The challenge is to manage the brand system to achieve clarity and synergy and to leverage brand assets into new markets and products.

Thus, subbrands can add clarity by augmenting the brand meaning. The Marriott subbrands such as Suites, Courtyard, Residence Inn, Resort Hotels, and Fairfield Inn all involve subbrands which provide clarity to their offerings and manage customer expectations. Smuckers Simply Fruit augmented the Smucker's quality and taste identity to include a fresh fruit association. Proctor \& Gamble introduced Sure ProStick to create male associations for the Sure brand.

A system of brands should be actively managed to gain synergy. Thus, the HewlettPackard (H-P) brand should enhance the Laserjet line by providing the endorsement of a respected, credible organisation and, conversely, the LaserJet success should reinforce and enhance the $\mathrm{H}-\mathrm{P}$ identity in part by enhancing H-P's image of being a successful leader. A branded feature or service such as H-P's Resolution Enhancement, Oral-B's Power Tip bristles, AT\&T's 'Your True Voice' and Hyatt's Business Portfolio can add credibility and substance to a brand.

Some brands have the potential to act as silver bullets, brands that can meaningfully influence the image of other brands. The Mazda Miata, the Dodge Viper, and the Ford Taurus all played silver bullet roles in that they were vehicles to change the image of Mazda, Dodge, and Ford. A health care programme with a reputation for being bureaucratic could use a nutrition and exercise programme for the elderly, given a brand name such as HeartClub, to help defuse the impersonal image. The San Jose Sharks major league hockey team plays a silver bullet role for San José, a city that is in the shadow of its neighbour San Francisco. The Sony Walkman supports the innovative, miniaturisation identity that is central to Sony.

In the attribute-fixation trap, strategic and tactical management of the brand becomes fixated on product or service attributes. Although an attribute-centred strategy is comfortingly logical, usually based on wellaccepted attribute-based research, and often effective, it can also have important limitations. Attributes often present fixed targets that are easy to duplicate or surpass. As Regis McKenna has noted - you can always be outspec'd. Perhaps worse, an attribute-based strategy can fail to differentiate if several competitors choose to focus on the same 'most important' attribute or benefit.

One way to avoid the attribute-fixation trap is to expand a brand's value proposi- 
tion to include emotional benefits and selfexpressive benefits. Emotional benefits focus on the potential of the brand to engender feelings such as safety (Volvo), confidence (Brooks Brothers), or health (Healthy Choice). Self-expressive benefits occur when the use of a brand serves to help express a person's own identity. A Harley owner may be expressing a desire to be free of schedules and a confining lifestyle. A baker or cook with a self-image of being the best may express his or her identity by buying first-class equipment for the kitchen.

Another way to broaden the brand concept is to look at the brand not only as a product or service but as an organisation and as a person. The brand-as-organisation perspective focuses on organisational attributes (such as being innovative, quality driven, customer driven, or environmentally conscious) that are created by the people, culture, values and programmes of the organisation. The Saturn brand from GM is a good example of an identity primarily based on an organisation - the Saturn values (building a world class economy car), programmes (eg the retailer low-pressure sales experience) and people (who visibly buy into the values). Organisational attributes are more enduring and more resistant to competitive claims, in part because it is much harder to copy an organisation than a product. It is harder to show that one's organisation is more innovative than to show that one's printer is faster.

The brand-as-person perspective suggests a brand identity that is richer, less sterile, and more interesting than attribute-based concepts. Like a person, a brand can be perceived as being upscale, competent, impressive, trustworthy, fun, active, humorous, casual, formal, youthful, or intellectual. For example, Saturn has the personality of a reliable friend who is fun-loving, down-toearth, and easy-going.
A brand personality can help provide a link to the customer in several ways. First, a brand personality can help create or explain a self-expressive benefit. Second, just as human personalities affect relationships between people, a brand personality can be the basis of a relationship between the customer and the brand. The friend relationship helps drive the Saturn identity and programme. Thus, Dell computer might be a professional who helps with the tough jobs, Levi a rugged outdoor companion, Mercedes an upscale, admired person, WordPerfect a competent, caring professional, and Hallmark a warm, emotional relative. Third, a brand personality may be a way to communicate a product attribute and thus contribute to a functional benefit. For example, the Michelin man represents both a product and personality that is strong and energetic.

The external perspective trap occurs when brand strategists (particularly those in the USA and Europe) assume that the only role of a brand identity is to influence customers. The orientation becomes entirely external. However, a brand identity can and should help an organisation understand its basic values and purpose. Because an effective identity is based in part on a disciplined effort to specify the strengths, values, and vision of the brand, it can provide a vehicle to communicate internally what the brand is about. Employees are more likely to make a vision happen if they understand and buy into that vision.

In most organisations, employees have a difficult time answering the question, "What does your brand stand for?' 'Achieving a 10 per cent increase in sales' (or profitability) - an all-too-typical response - is hardly inspiring. In firms with strong brands such as Saturn and Johnson \& Johnson, the response comes faster and with more substance from motivated (and even inspired) employees. Those in the Saturn organisation (including retailers and suppliers) know that Saturn stands for a world-class car and treat- 
ing customers with respect and as a friend. Those at Johnson \& Johnson know that its brand stands for trust and quality.

Broadening the concept of a brand. The overall theme here is that there is substantial value in broadening the concept of a brand - to consider its role in a larger brand sys- tem, to move beyond product attributes, and to view it as a device to improve internal as well as external communication.

David A. Aaker Editorial Board 